CERN-TH.7425/94

SWAT/93-94/39

hep-th/9409141

September 1994

\title{
THE EXACT MASS-GAP OF THE SUPERSYMMETRIC $O(N)$ SIGMA MODEL
}

\author{
Jonathan M. Evans* Timothy J. Hollowood** \\ CERN-TH, CH-1211 Geneva 23, Switzerland. \\ evansjm@surya11.cern.ch, hollow@surya11.cern.ch
}

\begin{abstract}
A formula for the mass-gap of the supersymmetric $\mathrm{O}(N)$ sigma model $(N>4)$ in two dimensions is derived: $m / \Lambda_{\overline{\mathrm{MS}}}=2^{2 \Delta} \sin (\pi \Delta) /(\pi \Delta)$, where $\Delta=1 /(N-2)$ and $m$ is the mass of the fundamental vector particle in the theory. This result is obtained by comparing two expressions for the free-energy density in the presence of a coupling to a conserved charge; one expression is computed from the exact S-matrix of Shankar and Witten via the the thermodynamic Bethe ansatz and the other is computed using conventional perturbation theory. These calculations provide a stringent test of the Smatrix, showing that it correctly reproduces the universal part of the beta-function and resolving the problem of CDD ambiguities.
\end{abstract}

CERN-TH.7425/94

September 1994

* Supported by a fellowship from the EU Human Capital and Mobility programme.

** On leave from: Department of Physics, University of Wales, Swansea, SA2 8PP, U.K. 


\section{Introduction}

There are many two-dimensional field theories which are quantum integrable and hence, following conventional wisdom, which are thought to be described by an exact factorizable S-matrix. Of particular interest are the theories which generate their mass dynamically, like the $O(N)$ sigma model, since they share many of the features of QCD in four dimensions. In general the exact S-matrices for these models are - if truth be told-conjectures which are postulated on the basis of symmetries and various physical properties which are encoded as axioms of S-matrix theory. The property of factorization is then enough in many cases to determine the S-matrix up to ambiguities of CDD type [1]. It is important to scrutinize these S-matrices and find ways of checking whether they do indeed describe the field theories for which they are designed.

Given a factorizable S-matrix, it is possible to find a set of integral equations, called the Thermodynamic Bethe Ansatz (TBA) equations, which determine the free-energy of the theory on a cylinder in the presence of a chemical potential which couples to a conserved charge in the model. It was realized some time ago, in the context of the $\operatorname{SU}(N)$ principal chiral models, that it is possible to use the TBA equations to extract the universal coefficients of the beta-function directly from the S-matrix $[2,3]$. The idea is to compare the free-energy extracted from the TBA equations at zero temperature (hence on the plane) to the same quantity evaluated in the asymptotic regime where the chemical potential is large and so perturbation theory is valid. Comparing these expressions provides a stringent test of the proposed S-matrix and also yields an exact value for the mass-gap of the theory, by which we mean the ratio of some chosen physical mass $m$ to the $\Lambda$-parameter of perturbation theory.

This strategy has been applied to a series of models: [4,5] for the $\mathrm{O}(N)$ sigma model; [6] for the $\mathrm{SU}(N)$ principal chiral model; [7] for the $\mathrm{SO}(N)$ and $\operatorname{Sp}(N)$ principal chiral models; [8] for the SU $(N)$ chiral Gross-Neveu models; [9] for the $\mathrm{O}(N)$ Gross-Neveu models; and [10] for integrable sigma models on an $\mathrm{SU}(2)$ group manifold with torsion. In each case the exact mass-gap was extracted and the S-matrix tested. It is perhaps significant that in each case the minimal S-matrix (the S-matrix with the minimum number of poles and zeros on the physical strip consistent with physical requirements) was found to be consistent. It is clearly very useful to have such exact results for mass gaps because they provide a remarkable opportunity to test the efficacy of lattice simulations or other nonperturbative approaches. In addition to this, however, the results of [4-10] provide valuable concrete illustrations of the correctness of conventional beliefs regarding the character of asymptotically-free theories with dynamical mass generation.

In this paper we shall apply the techniques described above to the supersymmetric $\mathrm{O}(N)$ sigma model $[11,12,13]$. The application of these methods to a supersymmetric 
integrable field theory raises some novel issues, as we shall see. In technical terms we must face a diagonalization problem for the TBA equations which does not arise in the purely bosonic case. In many respects the $\mathrm{O}(N)$ theories which we consider here are the simplest family of supersymmetric sigma models. There is another very well-known family of super sigma models based on $\mathbb{C P}^{n}$, which in fact exhibit extended $(N=2)$ supersymmetry, and which have a richer structure than the $\mathrm{O}(N)$ models at both the classical and quantum levels. These $\mathbb{C P}^{n}$ models give rise to a more complicated set of TBA equations and they will be treated in a sequel to this paper [14].

\section{The model and its S-matrix}

The lagrangian density of the supersymmetric $\mathrm{O}(N)$ model is [11]

$$
\mathcal{L}=\frac{1}{2 g}\left[\left(\partial_{\mu} n_{a}\right)^{2}+i \bar{\psi}_{a} \not \partial \psi_{a}+\frac{1}{4}\left(\bar{\psi}_{a} \psi_{a}\right)^{2}\right]
$$

where $n_{a}$ and $\psi_{a}$ are an $N$-component real scalar field and an $N$-component Majorana fermion respectively satisfying the constraints $n \cdot n=1$ and $n \cdot \psi=0$. We work throughout in two-dimensional Minkowski space and our conventions agree with those of $[11,15]$. We shall consider only the cases $N>4$ (the $\mathrm{O}(3)$ model fits more naturally into the family of $\mathbb{C P}^{n}$ theories discussed in [14] and the $\mathrm{O}(4)$ model is in fact equivalent to the principal chiral model based on $\mathrm{SU}(2)$ ). The theory (2.1) has a global $\mathrm{O}(N)$ symmetry and a global $N=1$ supersymmetry. Notice that the bosonic part of the theory is just the $\mathrm{O}(N)$ sigma model, the fermionic part is the $\mathrm{O}(N)$ Gross-Neveu model, and the coupling between the bosons and fermions is due solely to the constraint.

The two-loop beta-function for this model and the corresponding behaviour of the running coupling constant can be written

$$
\begin{aligned}
& \beta(g)=-\beta_{1} g^{2}-\beta_{2} g^{3}+\mathcal{O}\left(g^{4}\right), \\
& \text { so } \frac{1}{g(\mu / \Lambda)}=\beta_{1} \ln \frac{\mu}{\Lambda}+\frac{\beta_{2}}{\beta_{1}} \ln \ln \frac{\mu}{\Lambda}+\mathcal{O}\left(\frac{\ln \ln (\mu / \Lambda)}{\ln (\mu / \Lambda)}\right), \\
& \text { where } \beta_{1}=(N-2) / 2 \pi, \quad \beta_{2}=0 .
\end{aligned}
$$

Note that the first coefficient of the beta-function coincides with the result for the purely bosonic $\mathrm{O}(N)$ model, whereas the second coefficient vanishes, unlike the purely bosonic or Gross-Neveu cases. The values of both these coefficients can be deduced from general results [15] concerning supersymmetric sigma-models on locally symmetric spaces (see also [16] and references cited there for details of subsequent work). We see from the betafunction that the theory is asymptotically free with dynamical mass generation.

The integrability of the supersymmetric $\mathrm{O}(N)$ theory was studied over 16 years ago by Shanker and Witten [12] who, following [1], proposed a factorizable S-matrix to describe the 
scattering of the fundamental multiplet of particles in the model. It is expected that this fundamental multiplet will appear as a massive supersymmetric doublet which transforms as a vector under the $\mathrm{O}(N)$ symmetry. We will denote the corresponding quantum states by $|a, i, \theta\rangle$, where $i=0,1$ labels a boson, fermion respectively, $a$ is the $\mathrm{O}(N)$ vector index and $\theta$ is the rapidity of the particle, so that its velocity is $v=\tanh (\theta)$. The full spectrum of the theory will also contain bound states of this fundamental multiplet, but detailed knowledge of these will not be important for our purposes.

The $S$-matrix conjectured by Shankar and Witten has a very particular form in which the supersymmetric and $\mathrm{O}(N)$ degrees of freedom are factored. This means that the twobody $S$-matrix elements, from which all others follow, can be written [17]

$$
\left.\left\langle c, k, \theta_{2} ; d, l, \theta_{1} \text {, out }\right| a, i, \theta_{1} ; b, j, \theta_{2}, \text { in }\right\rangle=S_{\mathrm{SUSY}}\left(\theta_{1}-\theta_{2}\right)_{i j}^{k l} S_{\mathrm{GN}}\left(\theta_{1}-\theta_{2}\right)_{a b}^{c d} .
$$

Here the $\mathrm{O}(N)$ part is the factorizable S-matrix of the fundamental vector particle of the $\mathrm{O}(N)$ Gross-Neveu model $[1,18]$ :

$$
S_{\mathrm{GN}}(\theta)_{a b}^{c d}=Y_{1}(\theta)\left[\delta^{a d} \delta^{b c}-\frac{2 \pi i \Delta}{i \pi-\theta} \delta^{a b} \delta^{c d}-\frac{2 \pi i \Delta}{\theta} \delta^{a c} \delta^{b d}\right],
$$

where $\Delta=1 /(N-2)$ and the unitarizing-crossing scalar factor is $Y_{1}(\theta)=R_{1}(\theta) R_{1}(i \pi-\theta)$ with

$$
R_{1}(\theta)=\frac{\Gamma(-\Delta-i \theta / 2 \pi) \Gamma\left(\frac{1}{2}-i \theta / 2 \pi\right)}{\Gamma(-i \theta / 2 \pi) \Gamma\left(\frac{1}{2}-\Delta-i \theta / 2 \pi\right)} .
$$

The supersymmetric part of the S-matrix has the form

$$
S_{\mathrm{SUSY}}(\theta)=Y_{2}(\theta)\left(\begin{array}{cccc}
1+2 i \frac{\sin (\pi \Delta)}{\sinh \theta} & 0 & 0 & i \frac{\sin (\pi \Delta)}{\cosh (\theta / 2)} \\
0 & i \frac{\sin (\pi \Delta)}{\sinh (\theta / 2)} & 1 & 0 \\
0 & 1 & i \frac{\sin (\pi \Delta)}{\sinh (\theta / 2)} & 0 \\
-i \frac{\sin (\pi \Delta)}{\cosh (\theta / 2)} & 0 & 0 & -1+2 i \frac{\sin (\pi \Delta)}{\sinh \theta}
\end{array}\right) \text {, }
$$

in which the rows and columns are labelled in the order $(0,0),(0,1),(1,0),(1,1)$. In this case the scalar factor is $Y_{2}(\theta)=R_{2}(\theta) R_{2}(i \pi-\theta)$ where $^{1}$

$$
\begin{aligned}
& R_{2}(\theta)=\frac{\Gamma(-i \theta / 2 \pi)}{\Gamma\left(\frac{1}{2}-i \theta / 2 \pi\right)} \\
& \times \prod_{j=1}^{\infty} \frac{\Gamma(-\Delta-i \theta / 2 \pi+j) \Gamma(-i \theta / 2 \pi+\Delta+j-1) \Gamma^{2}\left(-i \theta / 2 \pi+j-\frac{1}{2}\right)}{\Gamma\left(-\Delta-i \theta / 2 \pi+j+\frac{1}{2}\right) \Gamma\left(-i \theta / 2 \pi+\Delta+j-\frac{1}{2}\right) \Gamma^{2}(-i \theta / 2 \pi+j-1)} .
\end{aligned}
$$

It is important that the ordering of the particles in the final state is taken so that the particle of rapidity $\theta_{2}$ is to the left of the particle with rapidity $\theta_{1}$; it is only this "modified"

1 When comparing with the expressions in [12] it is helpful to notice that we are including the simple pole term in the $\mathrm{O}(N)$ rather than in the supersymmetric factor; the opposite choice was made in [12] but the net results are obviously equivalent. 
S-matrix that displays the factorization of supersymmetric and bosonic degrees of freedom as described in [17].

The S-matrix (2.3) is a "minimal" expression in the sense that it has the minimum number of poles and zeros on the physical strip (the region $0 \leq \operatorname{Im}(\theta) \leq \pi$ ) consistent with the requirements of symmetry, the axioms of S-matrix theory, and the implementation of the bootstrap procedure. It is well-known, however, that solutions to these conditions are ambiguous precisely up to so-called CDD factors, which for the present model take the form

$$
\frac{\sinh \theta-i \sin (\pi \Delta(2-\alpha))}{\sinh \theta+i \sin (\pi \Delta(2-\alpha))} \cdot \frac{\sinh \theta-i \sin (\pi \Delta \alpha)}{\sinh \theta+i \sin (\pi \Delta \alpha)}
$$

where $0<\alpha<2$ is a constant. Multiplying (2.3) by any number of factors of this type does not introduce any additional poles on the physical strip and respects the internal consistency of the S-matrix. An important aspect of the results we shall obtain is that they will resolve this possible ambiguity in favour of the minimal choice (2.3). This is established by simply adopting the minimal S-matrix (2.3) and checking that the results derived from it agree exactly with perturbation theory, whereas any additional CDD factors would alter substantially the result of our calculation.

For completeness we mention how the entire spectrum of the model can be determined. The minimal S-matrix of the fundamental particles has a simple pole on the physical strip at $\theta=2 \pi i \Delta$ which corresponds to a bound state transforming in a reducible representation which is the sum of the antisymmetric tensor and singlet representations of $\mathrm{O}(N)$. Continuing the bootstrap in this way one finds a spectrum of bound-states which is identical to the $\mathrm{O}(N)$ Gross-Neveu model, namely $m_{r}=m \sin (\pi r \Delta) / \sin (\pi \Delta), 1 \leq r<(N / 2)-1$, apart from the fact that here each particle carries additional supersymmetric quantum numbers. We shall only require the S-matrix elements of the fundamental particle for our calculation.

\section{Coupling to a conserved charge}

To follow the logic of [4-10] one couples the theory to a background field $h$ via a conserved charge $Q$ corresponding to some generator of a global symmetry in the model. The field $h$ acts as a chemical potential for eigenstates of $Q$, and the idea is to compute the corresponding free-energy per unit volume $f(h)$ in the ultra-violet, large $h$, regime. This is clearly equivalent to calculating the ground state energy density of the system with the Hamiltonian modified from $H$ to $H-h Q$. In fact we are interested only in the finite difference $\delta f(h)=f(h)-f(0)$. The TBA equations which follow from the S-matrix in principle determine this quantity exactly, however one cannot usually solve them for all $h$. If the system is sufficiently simple, one can obtain an expansion valid in the asymptotic regime $h \gg m$, yielding a result of the form $\delta f(h)=h^{2} F_{1}(h / m)$. But $h \gg m$ is also exactly the regime in which conventional perturbation theory can be applied and such a calculation 
yields an expression of the form $\delta f(h)=h^{2} F_{2}(h / \Lambda)$. By equating $F_{1}(h / m)=F_{2}(h / \Lambda)$ one obtains a powerful check of the consistency of the proposed S-matrix with perturbation theory, and one extracts the mass-gap $m / \Lambda$.

A crucial part of this procedure is the precise choice of $Q$. The strategy followed in [4-10] is to choose $Q$ so that it has a unique largest eigenvalue, +1 say, corresponding to some unique fundamental particle state. Then one argues that for large $h$ only this particle state appears in the ground state of the new Hamiltonian $H-h Q$ and the TBA system is thereby reduced to a single integral equation. In fact this argument assumes that bound states which may have the same $Q$ eigenvalue will not contribute either, on the grounds that they will have a smaller charge/mass ratio. In principle this should follow from a rigorous analysis of the full TBA system, but in practice the complexity of the full system means that the assumption must usually be taken as a working hypothesis which is ultimately vindicated by the final results [4-10].

A novel feature of dealing with a supersymmetric system is that the best one can do is to choose $Q$ so that its largest eigenvalue picks out a supermultiplet of states rather than a single state (since $Q$ commutes with supersymmetry). In the present case we can choose, for example, a charge with

$$
Q_{12}=i, \quad Q_{21}=-i,
$$

and all other components zero. It is then exactly the doublet of states $(|1, j, \theta\rangle+$ $i|2, j, \theta\rangle) / \sqrt{2}$ which have eigenvalue +1 under $Q$ (whereas all the other eigenstates have eigenvalues 0 or -1 ). Our hypothesis is that only these particles will appear in the groundstate of the new Hamiltonian $H-h Q$. The scattering of these states amongst themselves is elastic in the space of $\mathrm{O}(N)$ quantum numbers but it is still non-diagonal in the supersymmetric subspace. The explicit S-matrix for these states is

$$
S(\theta)_{i j}^{k l}=U(\theta) S_{\mathrm{SUSY}}(\theta)_{i j}^{k l},
$$

where the scalar factor comes from the elastic scattering of the $\mathrm{O}(N)$ part of the S-matrix and has the form

$$
U(\theta)=\frac{\Gamma(1+i \theta / 2 \pi) \Gamma\left(\frac{1}{2}-i \theta / 2 \pi\right) \Gamma(1-\Delta-i \theta / 2 \pi) \Gamma\left(\frac{1}{2}-\Delta+i \theta / 2 \pi\right)}{\Gamma(1-i \theta / 2 \pi) \Gamma\left(\frac{1}{2}+i \theta / 2 \pi\right) \Gamma(1-\Delta+i \theta / 2 \pi) \Gamma\left(\frac{1}{2}-\Delta-i \theta / 2 \pi\right)} .
$$

As a result of the non-trivial scattering amongst the supersymmetric degrees of freedom we shall have to confront a set of two coupled TBA equations instead of a single integral equation as in [4-10].

Another important aspect of the choice of $Q$ is that it can drastically affect the nature of the expressions $F_{1}(h / m)$ and $F_{2}(h / \Lambda)$ which we are trying to calculate. Experience with other models [4-9] suggests that bosonic theories generally require perturbation theory to just one loop to extract the mass-gap (although the theories in [10] are an exception) whereas purely fermionic theories seem to require three loop calculations. Fortunately, we shall find that a one-loop calculation suffices for the choice of $Q$ given above. 


\section{Free-energy from perturbation theory}

The coupling to the charge $Q$ by means of the replacement $H \rightarrow H-h Q$ can be achieved at the lagrangian level by taking $\mathcal{L}$ and making the replacement $\partial_{0} \rightarrow \partial_{0}+$ $i h Q$ which resembles a covariant derivative. Having introduced this coupling, we wish to compute the free-energy to one-loop, which means that it is sufficient to expand the lagrangian to quadratic order in some set of independent fields. Since we are interested only in the difference $\delta f(h)=f(h)-f(0)$ we can also ignore any fields which do not couple to $h$ at this order. If we solve the bosonic constraint by writing $\left(n_{1}, n_{2}\right)=(\cos \theta, \sin \theta) \sqrt{1-\pi^{2}}$ where $\pi=\left(n_{3}, n_{4}, \ldots, n_{N}\right)$ then it is easy to see that the field $\theta$ decouples, as do all the fermionic degrees of freedom, and it suffices to consider the lagrangian

$$
\mathcal{L}_{1-\text { loop }}=\frac{1}{2 g}\left(\partial_{\mu} \pi\right)^{2}+\frac{h^{2}}{2 g}\left(1-\pi^{2}\right) .
$$

This leaves exactly the same calculation as encountered in the bosonic $\mathrm{O}(N)$ sigma model $[5]$.

Using dimensional regularization with the $\overline{\mathrm{MS}}$-scheme one finds the result

$$
\delta f(h)=-\frac{h^{2}}{2 g}+\frac{h^{2}}{8 \pi}(N-2)\left(1-\ln \left(h^{2} / \mu^{2}\right)\right)+\mathcal{O}(g)
$$

in terms of the running coupling $g\left(\mu / \Lambda_{\overline{\mathrm{MS}}}\right)$. We can use the fact that $\delta f(h)$ is a physical quantity, and therefore RG-invariant, to extract $\beta_{1}$ from the expression above and to check that it agrees with (2.2). (One cannot extract $\beta_{2}$ from this result alone because it is only valid to one-loop.) Now to compare with the TBA result we must substitute the explicit expression for the running coupling to two loops given in (2.2). For future reference we first write the result in a way which reveals the functional dependence on the coefficients of the beta-function:

$$
\delta f(h)=-h^{2} \frac{\beta_{1}}{2}\left[\ln \frac{h}{\Lambda_{\overline{\mathrm{MS}}}}-\frac{1}{2}+\frac{\beta_{2}}{\beta_{1}^{2}} \ln \ln \frac{h}{\Lambda_{\overline{\mathrm{MS}}}}+\mathcal{O}\left(\frac{\ln \ln \left(h / \Lambda_{\overline{\mathrm{MS}}}\right)}{\ln \left(h / \Lambda_{\overline{\mathrm{MS}}}\right)}\right)\right] .
$$

On taking the specific values of these coefficients given in (2.2) we obtain

$$
\delta f(h)=-(N-2) \frac{h^{2}}{4 \pi}\left[\ln \frac{h}{\Lambda_{\overline{\mathrm{MS}}}}-\frac{1}{2}+\mathcal{O}\left(\frac{\ln \ln \left(h / \Lambda_{\overline{\mathrm{MS}}}\right)}{\ln \left(h / \Lambda_{\overline{\mathrm{MS}}}\right)}\right)\right],
$$

which can be contrasted with the result for the bosonic $\mathrm{O}(N)$ sigma model (equation (18) of $[5])$. Notice that the absense of the $\ln \ln \left(h / \Lambda_{\overline{\mathrm{MS}}}\right)$ term is due to the vanishing of the second coefficient of the beta-function. We also remark that the number of terms in the expansion of $\delta f(h)$ in (4.4) will suffice to exact the mass-gap; this is directly related to the existence of the "tree-level" $\mathcal{O}(1 / g)$ term in (4.2). In contrast to this, for a fermionic model there is no tree-level contribution and a three-loop calculation is needed to extract the mass-gap. 


\section{Free-energy from the S-matrix}

We now write down the TBA equations for the model and find their solution in the limit $h \gg m$. Recall our hypothesis that with the coupling to the charge (3.1) only the states $(|1, j, \theta\rangle+i|2, j, \theta\rangle) / \sqrt{2}$ will contribute to the ground-state; this allows us to avoid the difficult problem of solving the full TBA equations including the $\mathrm{O}(N)$ magnon system. But these states are, after all, a supersymmetric multiplet and the scattering is non-diagonal in this subspace. Fortunately, the diagonalization of the relevant system of equations has been performed recently by Ahn [19] who exploited the equivalence of the problem to that of diagonalizing the transfer matrix of the eight vertex model at the free fermion point.

The set of equations derived in [19] relates the density of single particle states in rapidity space $\varrho(\theta)$ to the density of occupied single particle states $\sigma(\theta)$. The equations involve additional densities $P_{+}(\theta)$ and $P_{-}(\theta)$ corresponding to a "supersymmetric magnon":

$$
\begin{aligned}
& \varrho(\theta)=\frac{m}{2 \pi} \cosh \theta+\Psi * \sigma(\theta)+\frac{1}{2} \Phi *\left[P_{+}(\theta)-P_{-}(\theta)\right], \\
& P_{+}(\theta)+P_{-}(\theta)=\Phi * \sigma(\theta),
\end{aligned}
$$

where $f * g(\theta)=\int_{-\infty}^{\infty} d \theta^{\prime} f\left(\theta-\theta^{\prime}\right) g\left(\theta^{\prime}\right)$. The kernels appearing in (5.1) are

$$
\begin{aligned}
& \Psi(\theta)=\frac{1}{2 \pi} \operatorname{Im} \frac{d}{d \theta} \ln \left[\frac{U(\theta) Y_{2}(\theta)}{\sinh \theta}\right], \\
& \Phi(\theta)=\frac{1}{2} \cdot \frac{\sin (2 \pi \Delta)}{\cosh ^{2} \theta-\cos ^{2}(\pi \Delta)} .
\end{aligned}
$$

We can rewrite (5.1) in a more suggestive form by eliminating $P_{-}(\theta)$ from the first equation:

$$
\frac{m}{2 \pi} \cosh \theta=\varrho(\theta)-\Phi_{\mathrm{GN}} * \sigma(\theta)-\Phi * P_{+}(\theta)
$$

where one finds

$$
\Phi_{\mathrm{GN}}(\theta)=\frac{1}{2 \pi} \operatorname{Im} \frac{d}{d \theta} \ln U(\theta) .
$$

Notice that in this form the kernel which multiplies $\sigma(\theta)$ involves a contribution only from the Gross-Neveu part of the S-matrix due to the remarkable cancellation:

$$
\frac{1}{2 \pi} \operatorname{Im} \frac{d}{d \theta} \ln \left[\frac{Y_{2}(\theta)}{\sinh \theta}\right]-\frac{1}{2} \Phi * \Phi(\theta)=0 .
$$

The passage to the TBA equations proceeds in the usual manner [20]. At finite temperature $T$ we define the excitation energies of the particle $\epsilon(\theta)$ and the magnon $\xi(\theta)$ via

$$
\frac{\sigma(\theta)}{\varrho(\theta)}=\frac{1}{e^{\epsilon(\theta) / T}+1}, \quad \frac{P_{-}(\theta)}{P_{+}(\theta)}=e^{\xi(\theta) / T} .
$$


We shall only require the TBA equations at zero temperature with a chemical potential $h$ coupled to the particles. In this case $\epsilon\left( \pm \theta_{\mathrm{F}}\right)=0$, where $\theta_{\mathrm{F}}$ is the Fermi rapidity, and $\epsilon(\theta)$ is negative for $-\theta_{\mathrm{F}}<\theta<\theta_{\mathrm{F}}$. The free-energy per unit volume at $T=0$ is given by

$$
\delta f(h)=\frac{m}{2 \pi} \int_{-\theta_{\mathrm{F}}}^{\theta_{\mathrm{F}}} d \theta \epsilon(\theta) \cosh \theta,
$$

where $\epsilon(\theta)$ is the solution of the $T=0$ TBA equations:

$$
\begin{aligned}
\epsilon(\theta)-\Phi_{\mathrm{GN}} * \epsilon^{-}(\theta)-\Phi * \xi^{-}(\theta) & =m \cosh \theta-h, \\
\xi(\theta)-\Phi * \epsilon^{-}(\theta) & =0,
\end{aligned}
$$

and we have used the notation

$$
f^{ \pm}(\theta)= \begin{cases}f(\theta) & f(\theta) \stackrel{>}{<0} \\ 0 & \text { otherwise. }\end{cases}
$$

Notice that if we remove the term involving the magnon from the first TBA equation then it reduces to that encountered in $\mathrm{O}(N)$ Gross-Neveu model [9].

We must now solve (5.8) and we will implicitly assume that the solution is unique. The crucial observation is that $\Phi(\theta)$ is a positive kernel; hence the solution has $\xi^{+}(\theta)=0$ and $\xi^{-}(\theta)=\Phi * \epsilon^{-}(\theta)$ so that the two-dimensional system reduces to a single equation for $\epsilon(\theta):$

$$
\epsilon^{+}(\theta)+R * \epsilon^{-}(\theta)=m \cosh \theta-h .
$$

where the kernel is

$$
R(\theta)=\delta(\theta)-\Phi_{\mathrm{GN}}(\theta)-\Phi * \Phi(\theta) .
$$

This equation differs from that encountered in the $\mathrm{O}(N)$ Gross-Neveu model by the presence of the term involving $\Phi * \Phi(\theta)$ and we shall find that this drastically alters the nature of the solution.

To determine the behaviour of the solution in the regime $h \gg m$ we calculate the Fourier transform of the kernel in (5.10) and find

$$
\begin{aligned}
& R(\theta)= \\
& \int_{0}^{\infty} \frac{d \omega}{\pi} \cos (\omega \theta)\left[\frac{\cosh ((1-2 \Delta) \pi \omega / 2)}{\cosh (\pi \omega / 2)} e^{\Delta \pi \omega / 2}-\frac{\cosh ^{2}((1-2 \Delta) \pi \omega / 2)}{\cosh ^{2}(\pi \omega / 2)}\right] \\
& =\int_{0}^{\infty} \frac{d \omega}{\pi} \cos (\omega \theta) \frac{\cosh ((1-2 \Delta) \pi \omega / 2) \sinh (\pi \Delta \omega)}{\cosh ^{2}(\pi \omega / 2)} e^{\pi \omega / 2}
\end{aligned}
$$

The nature of the solution depends upon whether or not this Fourier transform vanishes at the origin [9]. We see here that the part that comes from the Gross-Neveu model does not vanish, but the Fourier transform of the full kernel, with the effect of supersymmetry included, does vanish at the origin. The solution for our model is therefore of the type encountered in the bosonic models described in [4-6] rather than the fermionic models. 
To find the first few terms in the expansion of the solution one has to write the Fourier transform of the kernel in the form $1 /\left(G_{+}(\omega) G_{-}(\omega)\right)$ where $G_{ \pm}(\omega)$ are analytic in the upper (lower) half planes with $G_{-}(\omega)=G_{+}(-\omega)$. This determines uniquely

$$
\begin{aligned}
G_{+}(\omega)= & \frac{\Gamma\left(\frac{1}{2}-i(1-2 \Delta) \omega / 2\right) \Gamma(1-i \Delta \omega)}{\Gamma^{2}\left(\frac{1}{2}-i \omega / 2\right)} e^{-\frac{1}{2} \ln (-i \Delta \omega)} \\
& \times e^{-i \omega\left(\frac{1}{2}-\Delta\right)\left(1-\ln \left(-i \omega\left(\frac{1}{2}-\Delta\right)\right)\right)-i \omega \Delta(1-\ln (-i \omega \Delta))+i \omega\left(1-\ln \left(-i \frac{1}{2} \omega\right)\right)} .
\end{aligned}
$$

Following the discussion in $[6]$, if $G_{+}(i \xi)$ has an expansion for small $\xi$ like

$$
G_{+}(i \xi)=\frac{k}{\sqrt{\xi}} e^{-a \xi \ln \xi}\left(1-b \xi+\mathcal{O}\left(\xi^{2}\right)\right)
$$

then the first few terms of the free-energy for $h \gg m$ are given by

$$
\begin{gathered}
\delta f(h)=-\frac{h^{2} k^{2}}{4}\left[\ln \frac{h}{m}+\ln \left(\frac{\sqrt{2 \pi} k e^{-b}}{G_{+}(i)}\right)-1+a\left(\gamma_{\mathrm{E}}-1+\ln 8\right)\right. \\
\left.+\left(a+\frac{1}{2}\right) \ln \ln \frac{h}{m}+\mathcal{O}\left(\frac{\ln \ln (h / m)}{\ln (h / m)}\right)\right] .
\end{gathered}
$$

Our kernel does indeed have an expansion of the form (5.14) with

$$
k=\frac{1}{\sqrt{\pi \Delta}}, \quad a=-\frac{1}{2}, \quad \frac{\sqrt{2 \pi} k e^{-b}}{G_{+}(i)}=\frac{\sin (\pi \Delta)}{\pi \Delta} e^{\gamma_{\mathrm{E}} / 2+\left(\frac{3}{2}+2 \Delta\right) \ln 2},
$$

and so the first few terms in the free-energy are

$$
\delta f(h)=-\frac{h^{2}}{4 \pi \Delta}\left[\ln \frac{h}{m}+\ln \left(\frac{\sin (\pi \Delta)}{\pi \Delta} 2^{2 \Delta}\right)-\frac{1}{2}+\mathcal{O}\left(\frac{\ln \ln (h / m)}{\ln (h / m)}\right)\right] .
$$

\section{Comparison and Conclusions}

Comparing (5.17) with (4.4) we see that the result from the TBA calculation correctly produces the universal coefficients of the beta-function and, furthermore, we extract the value for the mass-gap of the supersymmetric $\mathrm{O}(N)$ sigma model:

$$
\frac{m}{\Lambda_{\overline{\mathrm{MS}}}}=2^{2 \Delta} \cdot \frac{\sin (\pi \Delta)}{\pi \Delta}, \quad \Delta=\frac{1}{N-2}, \quad N>4
$$

It is interesting to compare this result with a calculation in the large $N$ limit. We find from above that $m / \Lambda_{\overline{\mathrm{MS}}}=1+(2 \ln 2) / N+\mathcal{O}\left(1 / N^{2}\right)$ which agrees with the large $N$ analysis in [21]. Unfortunately the conjecture for the mass-gap for all $N$ in [21] is not correct because it is based on a mistaken ansatz for the functional dependence on $\Delta$.

Our calculations provide convincing evidence that the S-matrix of Shankar and Witten does indeed describe the supersymmetric $\mathrm{O}(N)$ sigma model. In particular, as emphasized in [6], the addition of CDD factors of the form (2.8) to the S-matrix would change the kernel $R(\theta)$ and drastically alter the thermodynamics of the system, including the expression (5.17) for the free-energy, consequently destroying the remarkable agreement with the perturbative result. 


\section{References}

[1] A.B. Zamolodchikov and Al. B. Zamolodchikov, Ann. Phys. 120 (1979) 253

[2] A. Polyakov and P.B. Wiegmann, Phys. Lett. B131 (1983) 121

[3] P.B. Wiegmann, Phys. Lett. B141 (1984) 217

[4] P. Hasenfratz, M. Maggiore and F. Niedermayer, Phys. Lett. B245 (1990) 522

[5] P. Hasenfratz and F. Niedermayer, Phys. Lett. B245 (1990) 529

[6] J. Balog, S. Naik, F. Niedermayer and P. Weisz, Phys. Rev. Lett. 69 (1992) 873

S. Naik, Nucl. Phys. B (Proc. Suppl.) 30 (1993) 232

[7] T.J. Hollowood, Phys. Lett. B329 (1994) 450

[8] P. Forgacs, S. Naik and F. Niedermayer, Phys. Lett. B283 (1992) 282

[9] P. Forgács, F. Niedermayer and P. Weisz, Nucl. Phys. B367 (1991) 123

[10] J.M. Evans and T.J. Hollowood, Integrable theories that are asymptotically CFT, Preprint CERN-TH.7293/94, SWAT/93-94/32, hep-th/9407113

[11] E. Witten, Phys. Rev. D16 (1977) 2991

[12] R. Shankar and E. Witten, Phys. Rev. D17 (1978) 2134

[13] P. DiVecchia and S. Ferrara, Nucl. Phys. B130 (1977) 93

[14] J.M. Evans and T.J. Hollowood, The exact mass-gap of the supersymmetric $\mathbb{C P}^{n}$ sigma model, preprint CERN-TH.7426/94, SWAT/93-94/42

[15] L. Alvarez-Gaumé, D.Z. Freedman and S.K. Mukhi, Ann. Phys. 134 (1981) 85

[16] M.T. Grisaru, A.E.M. de Ven and D. Zanon, Nucl. Phys. B277 (1986) 388; 409

[17] K. Schoutens, Nucl. Phys. B344 (1990) 665

[18] A.B. Zamolodchikov and Al.B. Zamolodchikov, Nucl. Phys. B133 (1978) 525 M. Karowski and H.J. Thun, Nucl. Phys. B190 (1981) 61

[19] C. Ahn, Thermodynamics and form factors in supersymmetric integrable theories, Trieste preprint IC/93/144, hep-th/9306146

[20] E.H. Lieb and W. Liniger, Phys. Rev. 130 (1963) 1605

A1.B. Zamolodchikov, Nucl. Phys. B342 (1990) 695

[21] J.A. Gracey, Phys. Lett. B298 (1993) 116 